\title{
Advanced D-InSAR Techniques Applied to a Time Series of Airborne SAR Data
}

\author{
Pau Prats, Rolf Scheiber \\ and Alberto Moreira \\ German Aerospace Center (DLR) \\ Microwaves and Radar Institute \\ Wessling D-82234, Germany \\ Email: Pau.Prats@dlr.de
}

\author{
Andreas Reigber \\ Berlin University of Technology \\ Computer Vision and Remote Sensing Dept. \\ Berlin D-10587, Germany \\ Email: anderl@cs.tu-berlin.de
}

\author{
Jordi J. Mallorqui \\ Universitat Politècnica de Catalunya \\ Remote Sensing Laboratory \\ Signal Theory and Communications Dept. \\ Barcelona E-08034, Spain \\ Email: mallorqui@tsc.upc.edu
}

\begin{abstract}
This paper presents airborne differential SAR results using a stack of 14 images, which were acquired by the Experimental SAR (E-SAR) system of the German Aerospace Center during a time span of only two and a half hours. An advanced differential technique is used to retrieve the error in the digital elevation model and the temporal evolution of the deformation for every coherent pixel in the image. The two main limitations in airborne SAR processing are analyzed, namely the existence of residual motion errors (inaccuracies in the navigation system in the order of $1-5 \mathrm{~cm}$ ), and the accommodation of the topography and the aperture during processing. The SAR focusing chain to process the data is also presented, together with the modifications in the differential processor to deal with the remaining baseline error. The detected deformation of a corner reflector and of several agricultural fields allows validating the proposed techniques.
\end{abstract}

\section{INTRODUCTION}

Differential synthetic aperture radar interferometry (DInSAR) has become a powerful tool to measure deformation phenomena at a large scale. Very high accuracy in the order of the wavelength can be attained by exploiting the coherent nature of SAR systems. Indeed, differential SAR interferometry using a spaceborne platform is already a quite established technique. The ideal stable trajectory of a satellite ensures the SAR processor will be able to properly focus the data without introducing undesired artifacts. Also, the fact that a large stack of images are available has been of great help to develop several advanced D-InSAR (ADInSAR) techniques. However, the airborne case is completely the opposite. First, there exist so far a few differential data sets with in-situ measurements to be able to validate results. But more importantly, the data processing becomes a challenge, since it is subject to the limitations imposed by motion compensation (MoCo). The fact that the platform is not following an ideally rectilinear trajectory arises several drawbacks that must be considered if accuracy is a priority. However, the advantages an airborne platform can offer are quite appealing: flexibility in sense of spatial resolution, used wavelength, and data acquisition (short revisit time).

The limitations existing in airborne repeat-pass interferometry are mainly two. The first limitation, addressed in Section II$\mathrm{A}$, is the fact that a reference height for the whole image must be assumed during motion compensation if efficient Fourierbased processors want to be used. The second, and most important, is the presence of residual motion errors (RME), i.e. inaccuracies in the measurements of the antenna positions in the order of $1-5 \mathrm{~cm}$. Section II-B addresses this topic.

The processing chain to focus the data is described in Section II-D, where a detailed description is given in order to deal with the commented limitations. Section III comments the advanced D-InSAR algorithm used to obtain the temporal evolution of the deformation and the modifications needed in the ADInSAR processing to consider RME. Finally, Section IV presents some results with data acquired by the Experimental SAR (E-SAR) system of the German Aerospace Center (DLR). Data were acquired the same day during only two and a half hours. However, results show some deformation in several agricultural fields, probably due to a change in soil moisture. The deformation in one corner reflector that was moved on purpose during the experiment is also retrieved.

\section{Motion COMPENSATION}

The real challenge in airborne SAR processing is motion compensation, above all in differential SAR interferometry. To reach millimeter accuracy at L-band the phase accuracy must be better than $3^{\circ}$. This implies a very accurate motion compensation scheme must be used to properly deal with the non-ideal trajectory of the platform. Hence, one must consider two important limitations: the unknown topography in the scene under observation, and the existence of RME.

\section{A. Topography- and Aperture-Dependent MoCo}

It has already been shown that conventional motion compensation algorithms are limited to obtain accurate airborne repeat-pass interferometric products. In particular, the unknown topography and the angle-dependence of the correction need to be considered. Several efficient topographyand aperture-dependent (TAD) algorithms have been recently proposed [1], of which SATA is used in the presented results. The amount of degradation in the image if no TAD algorithm is applied depends mainly on the topographic variations within the scene and the magnitude of the platform deviations. The main effects are phase errors and the azimuth shift of the 
impulse response function (IRF). Hence, such errors will result in a degraded interferogram, above all in repeat-pass interferometric systems, where track deviations are not correlated.

An important key point is the information content of an interferogram after processing master and slave images with a TAD algorithm. In [1] it is shown that the residual phase, i.e. the phase that remains after subtracting the synthetic phase obtained from the external DEM, is indeed proportional to the real baseline and the DEM error. Note that the subtraction of the synthetic phase is needed in order to take out the information introduced during motion compensation.

\section{B. Residual Motion Errors}

The absolute positioning accuracy of current navigation systems using inertial navigation systems and differential GPS is about $1-5 \mathrm{~cm}$. Therefore, the navigation data used during motion compensation can have residual motion errors, resulting mainly in phase errors and azimuth impulse response shifts. Although such errors will not degrade the high azimuthal resolution capability, some applications like repeat-pass interferometry or differential interferometry can be strongly limited by it. Consequently, some procedure is needed to estimate the remaining trajectory deviations. Several algorithms have been proposed in the literature, of which the multi-squint technique [2] has been used to estimate RME in the results of Section IV. This particular approach is based on the estimation of the azimuth coregistration offsets between multiple looks of an interferometric pair. The coregistration offsets are related to the derivative of RME, so that after a proper scaling and integration, the time-varying baseline error can be retrieved. The estimated baseline error can be used to update the tracks of the slave image to reprocess it, so that afterwards both master and slave images will have the same RME of the master image, hence cancelling out after interferogram generation.

\section{Coupling Between Unknown Topography and RME}

Up to know, the two main limitations, i.e. unknown topography during motion compensation and the estimation of RME (or baseline error), have been tackled separately. However, a coupling between them is sure to arise due to the fact that both have similar effects in the retrieved interferogram, mainly azimuth coregistration offsets. This can become a problem if time-varying baseline error estimation techniques based on the estimation of coregistration offsets are used, which is the case in the presented results. Therefore, it should be expected the retrieved baseline error to be biased. It is easy to foresee that a linear variation of the phase error through the synthetic aperture due to unknown topography introduces an undesired azimuth shift of the impulse response. Considering the high sensitivity of multi-squint to estimate coregistration errors, the induced coregistration error due to unknown topography can reach critical values that will bias the baseline error estimation. Hence, some procedure is needed to remove the remaining baseline error. In the presented case, this is done during the ADInSAR processing, as commented in Section III.

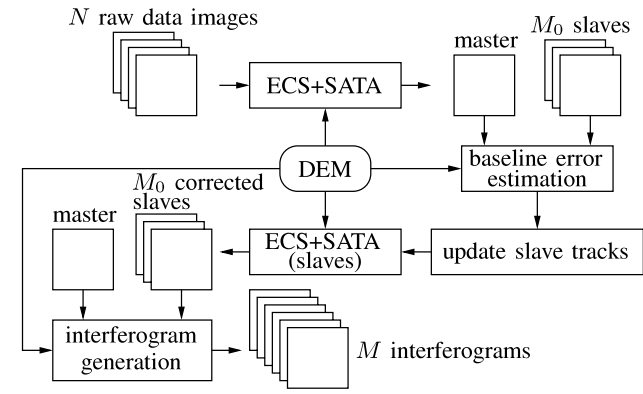

Fig. 1. Block diagram of the used processing chain.

\section{Implemented SAR Processing Chain}

The processing chain used to focus the data shown in Section IV is depicted in Fig. 1. In the present case, the idea is to generate $M$ interferograms from a set of $N$ acquisitions. Instead of generating $M_{0}=N-1$ interferograms, i.e. all slaves respect to a master image $I_{0}$, several interferograms can be generated as carried out in several advanced D-InSAR applications [3], so that $M$ is generally greater than $M_{0}$. The differential processor will later on retrieve the deformation for each individual acquisition. Once the SAR focusing is carried out for each image using ECS and SATA, multi-squint and the model-based integration proposed in [2] are used to estimate the baseline error for the first $M_{0}$ interferograms. Several iterations are performed with multi-squint to improve the estimation. After the last iteration, constant and linear terms of the baseline error, which cannot be estimated by means of multi-squint, are estimated using the external DEM as described also in [2]. The slave images are then reprocessed after updating their tracks with the full estimation of the baseline error. At this point, all the images have the residual motion error of image $I_{0}$. Consequently, any combination between them will result in a RME-free interferogram. Afterward, the interferometric processor carries out the typical steps: range interpolation, spectrum filtering, interferogram generation, coherence estimation, phase unwrapping and absolute calibration using a corner reflector. Once the interferometric processing of all $M$ interferograms is finished, the data are prepared to be processed by the advanced D-InSAR processor.

\section{AdVANCED AIRBORNE D-INSAR}

There exist several techniques to retrieve the deformation temporal evolution in a stack of images. The differential technique presented by Berardino et al. [3] has been selected to process the data of Section IV. Prior to starting the algorithm, it is assumed that the residual phases have been unwrapped and calibrated respect to one pixel whose deformation is known (usually a highly coherent pixel without deformation). This allows making a pixel-by-pixel temporal analysis. In a first step, the DEM error and the mean deformation velocity are estimated using a least-squares (LS) estimation. Once the DEM error and the mean deformation velocity have been estimated, they are subtracted to each interferogram. Next step is to find the deformation for each image, which is 
solved via single value decomposition (SVD). The problem with spaceborne data is that the atmospheric artifacts are still inside the estimated displacements. The filtering to estimate them is based on the fact that the atmospheric signal phase component is characterized by a high spatial correlation but exhibits a significantly low temporal correlation. Accordingly, the undesired atmospheric phase component can be estimated after the SVD as follows: first, the low-pass component of the deformation signal already estimated is removed. Afterwards, the atmospheric phase component is detected as the result of the cascade of a low-pass filtering step, performed in the twodimensional spatial domain, and a high-pass filtering operation with respect to the time variable. Once the atmospheric phase component has been evaluated, it is finally subtracted from the estimated phase signal.

However, the airborne case has to further deal with the presence of residual motion errors. The fact that RME might have not been properly estimated and corrected (as noted in Section II-C) implies some kind of filtering should be carried out to remove them from the differential interferograms. Furthermore, the atmospheric component cannot be neglected, even at L-band [4]. This filtering can be carried out at the same point as when the atmosphere is estimated in the spaceborne case, i.e. after the SVD estimation and having subtracted the previously estimated mean deformation velocity. The contribution of baseline errors and atmospheric artifacts can be described as

$$
\begin{aligned}
\phi_{\mathrm{err}}\left(t_{i}, x, r\right) & =\frac{4 \pi}{\lambda} \epsilon_{\mathrm{rme}}+\frac{4 \pi}{\lambda} \epsilon_{\mathrm{atm}} \\
& =\frac{4 \pi}{\lambda} \cdot\left(\epsilon_{\mathrm{y}}(x) \sin \theta(r)-\epsilon_{\mathrm{z}}(x) \cos \theta(r)\right) \\
& +\frac{4 \pi}{\lambda} 10^{-6} \cdot \int_{0}^{H} \frac{N(x, r, h)}{\cos \theta(r)} d h,
\end{aligned}
$$

where the baseline error $\epsilon_{\mathrm{rme}}$ is just the projection in LOS of the individual unknown horizontal $\epsilon_{\mathrm{y}}$ and vertical $\epsilon_{\mathrm{z}}$ displacements, and $N$ is the scaled-up refractivity equal to $N=(n-1) \cdot 10^{6}$, being $n$ the refractive index of the medium. Note that the slant-atmospheric delay is inversely proportional to the cosine of the look angle [4] and has a high spatial correlation. A possible approach to estimate these two components is to perform a LS estimation for every azimuth position $x$ to obtain the remaining baseline error using the same model as in [2]. After subtracting the estimated baseline error to the image, a large low pass filtering can be applied to estimate the atmospheric component. In the airborne geometry, the look angle changes considerably along the scene (ca. $20^{\circ}-60^{\circ}$ ). Therefore, in order to consider the look angle dependence, data should be first multiplied by $\cos \theta(r)$. Now the large low pass filter can be applied to the image to retrieve the atmospheric component. Finally, the estimated atmospheric component is subtracted from the data after dividing it by $\cos \theta(r)$. After the correction of baseline errors and atmosphere, a further HP filtering in time reduces the influence of these two effects, which are subtracted from the deformation series in the last step of the algorithm.
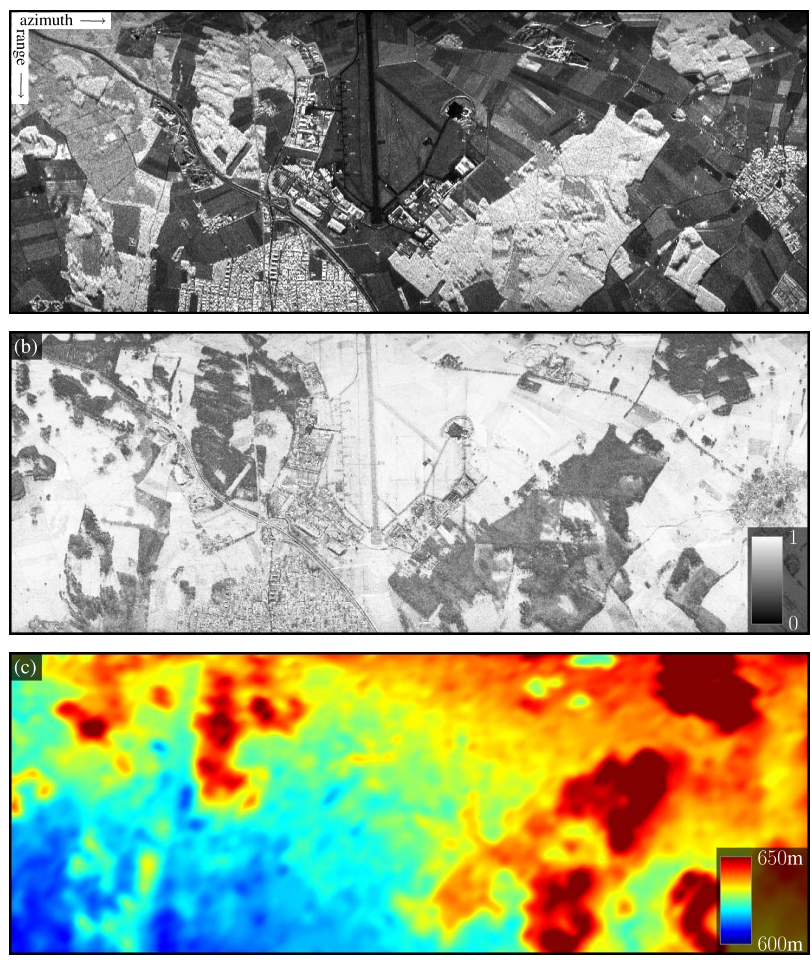

Fig. 2. (a) Reflectivity image of the observed scene, (b) Mean coherence for all 27 interferograms, and (c) SRTM DEM used during the processing.

\section{EXPERIMENTAL RESULTS}

A total of 14 images were acquired by the E-SAR system of DLR during a time span of only two and a half hours at L-band ( $\sim 11$ min between each acquisition), in the test site of Oberpfaffenhofen, Germany. The data acquisition took place on May $11^{\text {th }} 1998$ in order to carry out the first tomographic experiment with a SAR system [5]. This same data set has been used to analyze the performance and limitations of advanced D-InSAR techniques when working with airborne data. With 14 images, up to 91 interferograms can be formed. However, a maximum baseline of $50 \mathrm{~m}$ has been imposed in order to keep a large number of coherent points without much spectral filtering, resulting in a total number of 27 interferograms. Fig. 2(a) shows the reflectivity image of the observed scene. The multilook applied to the interferograms and equal to the window for coherence estimation is of $4 \times 4$ pixels, so that the image spacing is about $6 \mathrm{~m} \times 6 \mathrm{~m}$. Those pixels having a coherence larger than 0.8 in at least $50 \%$ of the interferograms have been selected. Also, the mask generated by the region growing algorithm for each interferogram has been used to discard pixels not properly unwrapped. Fig. 2(b) shows the mean coherence of all 27 interferograms, while Fig. 2(c) shows the SRTM DEM used during motion compensation, which has a nominal resolution of $90 \mathrm{~m} \times 90 \mathrm{~m}$.

All residual interferograms have been calibrated using a corner reflector next to the runway. After applying a LS estimation to each pixel, the DEM error and the mean deformation velocity maps are obtained. Fig. 3 shows these two 

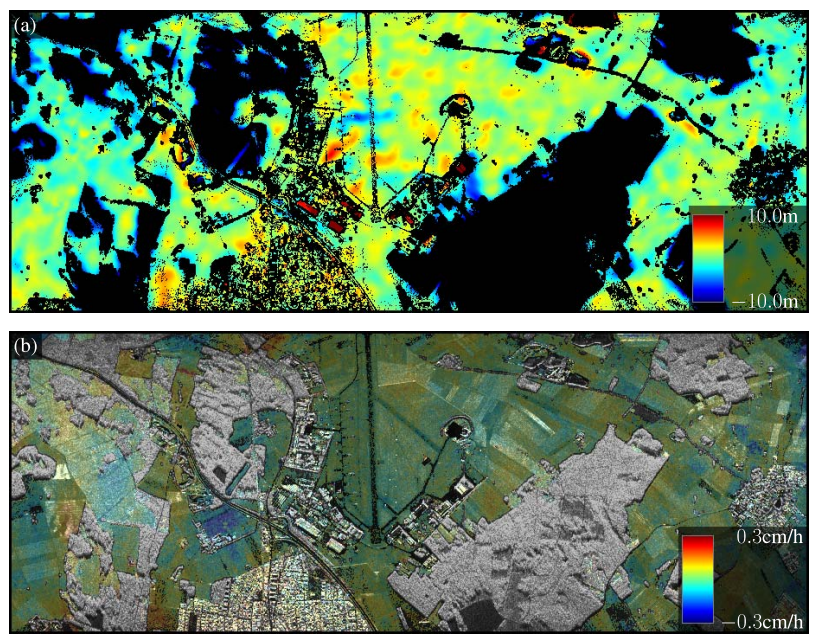

Fig. 3. Estimated (a) DEM error, and (b) mean deformation velocity in LOS with overlayed reflectivity. Masked values in black.

products. Some deformation is measured in a mobile corner reflector, which was indeed moved on purpose during the data take. Unexpectedly, some geometrical shapes show quite clearly all around the image and they do not seem to be a processing artifact. In fact, it can be noted how these shapes correlate well with the shapes of the agricultural fields. The most plausible explanation for these effects is that the soil moisture content changed, similarly as reported in [6]-[8]. After subtracting the DEM error, the SVD approach is applied to obtain the time sequence deformation of images. Then, the filtering commented in Section III is carried out to estimate the remaining baseline error and the atmosphere. The estimated atmospheric component is very small in this particular data set, with a standard deviation of only $0.7 \mathrm{~mm}$ in all images. In fact, most probably the estimated atmospheric component is due to noise. Fig. 4 shows the deformation at some time instants, where again it can be noted how the detected deformation areas correlate well with the shape of agricultural fields.

\section{CONCLUSiON}

This paper has shown the potential of airborne platforms to retrieve differential interferometric products, presenting results with a large stack of images and advanced D-InSAR techniques. The processing strategy to focus the data has been expounded, emphasizing the limitations in airborne systems. In particular, residual motion errors become the main limitation. The multi-squint technique used to estimate the baseline error can lead to a bias as a consequence of the undesired shift of the IRF due to errors in the external DEM. Therefore, a solution is proposed during the ADInSAR processing to estimate the remaining baseline error and hence reach very good accuracy in the estimation of the deformation. Besides the detection of a corner reflector that was moved on purpose, it has been possible to detect some deformation of just a few millimeters in several agricultural fields, probably due to a change in soil moisture during the data take. The high correlation between the shapes of the deformation areas and the agricultural fields
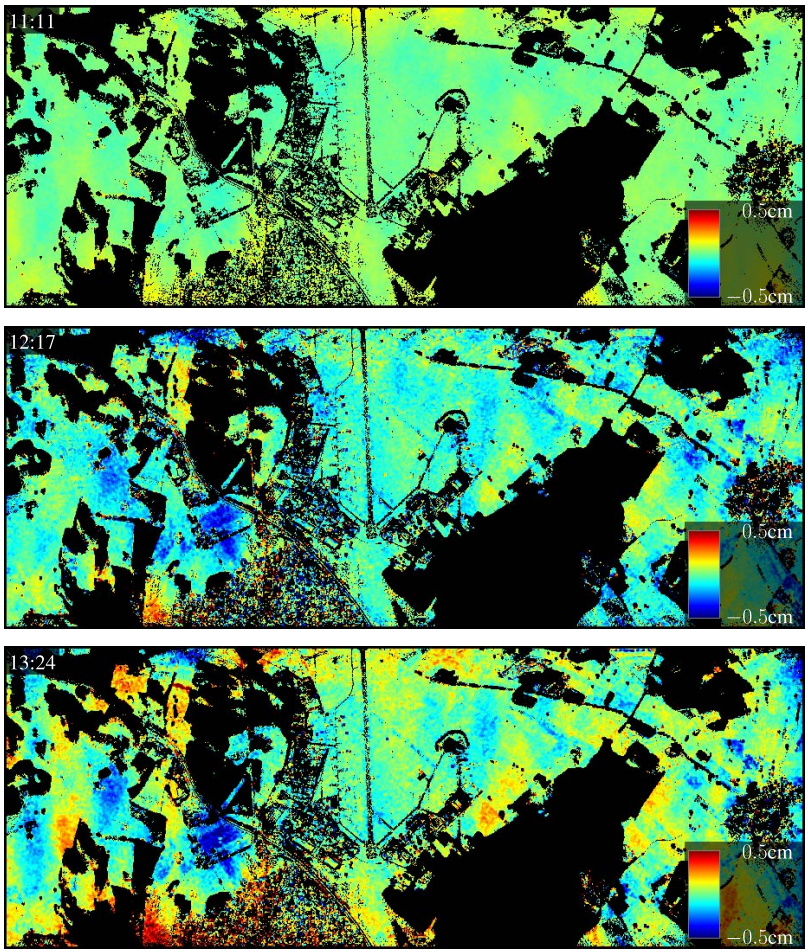

Fig. 4. Three images of the deformation evolution in LOS, with the local time of each image acquisition on the top-left corner. Acquisition started at 11:00 and finished at 13:24.

allows validating the presented results. Unfortunately, no insitu measurements are available. Ideally, a proper validation of the proposed techniques should be carried out by performing a campaign over a scenario with in-situ measurements.

\section{ACKNOWLEDGMENTS}

This work has been supported in part by the Spanish MCYT and EU FEDER funds under project TEC2005-068631-C02-01.

\section{REFERENCES}

[1] P. Prats, K. A. C. de Macedo, A. Reigber, R. Scheiber, and J. J. Mallorqui, "Comparison of topography- and aperture dependent motion compensation algorithms for airborne SAR,' IEEE Geosci. Remote Sensing Lett., vol. 4, no. 3, July 2007.

[2] A. Reigber, P. Prats, and J. J. Mallorqui, "Refined estimation of timevarying baseline errors in airborne SAR interferometry," IEEE Geosci. Remote Sensing Lett., vol. 3, no. 1, pp. 145-149, Jan. 2006.

[3] P. Berardino, G. Fornaro, R. Lanari, and E. Sansosti, "A new algorithm for surface deformation monitoring based on small baseline differential SAR interferograms," IEEE Trans. Geosci. Remote Sensing, vol. 40, no. 11, pp. 2375-2383, Nov. 2002.

[4] R. F. Hanssen, Radar Interferometry. Data Interpretation and Error Analysis. The Netherlands: Kluwer Academic Publishers, 2001.

[5] A. Reigber and A. Moreira, "First demonstration of airborne SAR tomography using multibaseline L-band data," IEEE Trans. Geosci. Remote Sensing, vol. 38, no. 5, pp. 2142-2152, Sept. 2000.

[6] A. K. Gabriel, R. M. Goldstein, and H. A. Zebker, "Mapping small elevation changes over large areas: Differential radar interferometry," Journal of Geophysical Research, vol. 94, pp. 9183-9191, 1989.

[7] M. Nolan and D. R. Fatland, "Penetration depth as a DInSAR observable and proxy for soil moisture," IEEE Trans. Geosci. Remote Sensing, vol. 41, no. 3, pp. 532-537, Mar. 2003.

[8] A. Reigber and R. Scheiber, "Airborne differential SAR interferometry: First results at L-band," IEEE Trans. Geosci. Remote Sensing, vol. 41, no. 6, pp. 1516-1520, June 2003. 